This item was submitted to Loughborough's Research Repository by the author.

Items in Figshare are protected by copyright, with all rights reserved, unless otherwise indicated.

\title{
Electrically induced heat dissipation in metallized film capacitors
}

PLEASE CITE THE PUBLISHED VERSION

PUBLISHER

(C) IEEE

VERSION

VoR (Version of Record)

LICENCE

CC BY-NC-ND 4.0

\section{REPOSITORY RECORD}

Kong, Michael G., and Yuen-Pen Lee. 2019. "Electrically Induced Heat Dissipation in Metallized Film Capacitors". figshare. https://hdl.handle.net/2134/5252. 
This item was submitted to Loughborough's Institutional Repository (https://dspace.lboro.ac.uk/) by the author and is made available under the following Creative Commons Licence conditions.

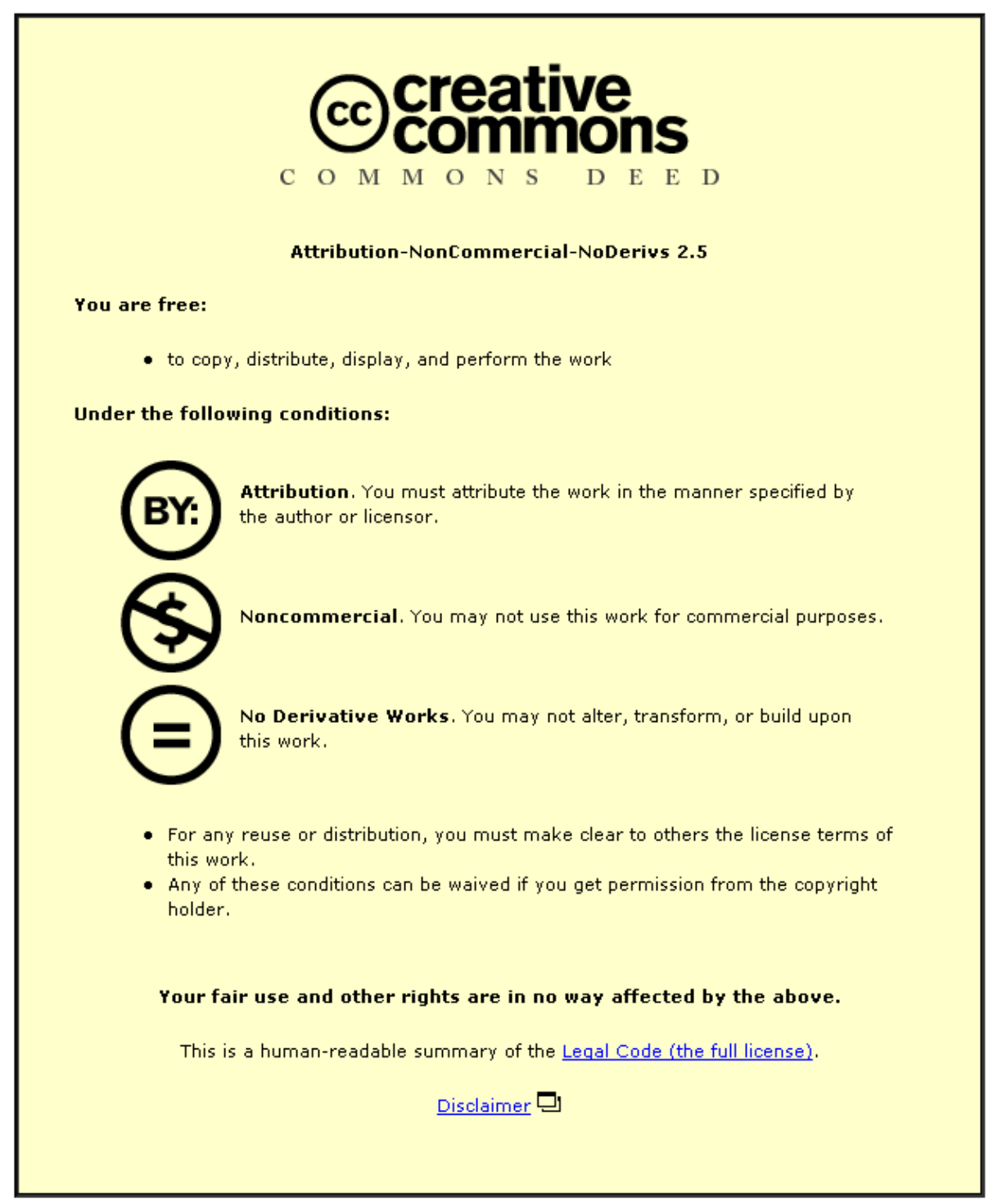

For the full text of this licence, please go to: http://creativecommons.org/licenses/by-nc-nd/2.5/ 


\title{
Electrically Induced Heat Dissipation in Metallized Film Capacitors
}

\author{
M. G. Kong and Y. P. Lee \\ Plasma and Pulsed Power Group \\ Department of Electronic and Electrical Engineering \\ Loughborough University, Loughborough, Leicestershire LE11 3TU, UK
}

\begin{abstract}
Elevated temperature is a key aging factor for metallized polymer film capacitors with the capacitor life expectancy halved with every $8^{\circ} \mathrm{C}$ of temperature rise. For film capacitors in service, both application of a time-varying external voltage and the extinction of internal breakdown events can deposit significant heat onto the capacitor structure leading to an undesirable temperature rise. Often such heat generation is localized spatially and the resulting temperature gradient enhances the probability of subsequent breakdowns. To facilitate a thorough and generic understanding, a thermal model of metallized film capacitors is developed to simulate the dynamics of heat generation and transfer under the switching impact of an external voltage. Thermal processes in solids (polymer layers, electrode coating, and zinc-spray segment) are modeled with conduction mechanism, whereas convection mechanism is considered for silicone oil and air. Heat generation is induced either by ohmic losses of the externally applied electric field, or by internal breakdown events within the metallized film capacitor structure. This allows a seamless interface between the heat transfer model and the equivalent circuit based field calculation model. Based this electrothermal model, numerical examples are used to study temperature distribution within film capacitors and then to examine the likelihood of localized temperature hot-spots that may lead to spatially preferential breakdowns.
\end{abstract}

Index Terms - Film capacitors, breakdown, heat transfer, film metallization.

\section{INTRODUCTION}

$\mathrm{F}$ OR numerous industrial applications of metallized film capacitors, there has been a fundamental need to improve their energy density within an increasingly compact housing. Inevitably this leads to smaller and smaller design margin with which insulation integrity and system reliability must be ensured. To this end, it is vital to gain a thorough and quantitative understanding of failure mechanisms in metallized film capacitors. This has, over the past three decades, led to wide-ranging investigations into a number of key aging factors [1-3], for example electrical, thermal, and chemical properties of capacitor dielectrics $[4,5]$ as well as effects of their material degradation under mechanical [6], electrical [7], and thermal stresses [8]. Extensive studies have also been conducted to establish the effects of the electric and chemical properties of liquid impregnants $[9,10]$, and waveforms and peak values of electric stress [11-13]. These studies are

Manuscript received on 4 October 2002, in final form 27 July 2004. predominately empirical in their approaches, and their findings have been responsible for the establishment of an invaluable pool of current test methodologies and design principles for modern metallized film capacitors.

One common feature of the current analysis and design techniques for metallized film capacitors is that they are not sufficiently generic having been developed through largely empirical routes. As a result the process of employing them to evaluate a potentially beneficial design modification is expensive and time-consuming requiring almost invariably extensive accelerated life tests that last typically 4-6 months for each possible combination of key system parameters (e.g. voltage stress and temperature). This has added significantly to development cost of future metallized film capacitor and has been an important factor in the slowed progress of the film capacitor technology over the past five years. Clearly what is much desired is a theoretical capability that can model adequately key dynamic processes in metallized film capacitors thus providing generic insight into key processes of capacitor opera- 
tion. This would allow at least a qualitative evaluation of the potential pros and cons of a new capacitor design before expensive and time-consuming accelerated life tests are committed.

Dynamic evolution of metallized film capacitors under their operational conditions is however extremely complex with inter-tangled and interacting physical, chemical, mechanical, and breakdown-discharge related processes. This partly explains the current lack of viable theoretical models of metallized film capacitors. Similarly the obvious difficulty of accounting for all relevant capacitor processes in an "all-in-one" global model favors a modular approach in which each key capacitor process (e.g. electrical, thermal, and discharge related) is treated with its own model before a global model interlacing all modular models together is developed $[14,15]$. This modular strategy has seen the development of simple theoretical tools that can compute efficiently the electric field distribution in film capacitors and its temporal evolution [15-17], and simulate accurately the dynamic evolution of surface discharges [18, 19]. Adopting the same strategic approach, this paper reports the development of a theoretical model to study thermal processes in polymeric film capacitors.

Thermal aging is one of the main failure mechanisms in film capacitors [2]. It is known that elevated temperature can considerably reduce life expectancy of metallised polymer film capacitors. For film capacitors in service, both the application of a time-varying external voltage and the extinction of internal breakdown events can release significant heat onto the capacitor structure, thus leading to an undesirable temperature rise. Often such heat generation is localized spatially and the resulting temperature gradient enhances the probability of subsequent breakdowns. In the temperature range from 40 to $65^{\circ} \mathrm{C}$, experimental data in [3] show that the life of metallised film capacitors is decreased by a factor of two for every $8^{\circ} \mathrm{C}$ of temperature increase. This highlights the need to understand heat generation and temperature distribution in film capacitors. In this paper, we will discuss the development of an electrothermal model for metallized film capacitors. Our objective is to provide a quantitative description of the time-dependent temperature distribution in metallised polymer film capacitors under selected heat generation conditions. In particular we will consider heat generation by ohmic losses induced by the externally applied electric field, which can be modeled from the equivalent circuit model described previously $[16,17]$. Thermal processes in solids (polymer layers, electrode coating, and zinc spray) are modeled mainly with heat conduction mechanism, whereas heat convection mechanism is considered for silicone oil and air. The heat transfer model interfaces with the equivalent circuit based field computation model. Using this electrothermal model, numerical examples are employed to study temperature distribution within film capacitors and then to examine the likelihood of localized temperature hot spots that may lead to spatially preferential breakdowns. It should be emphasized that under the conditions of low micro-discharge activities pure thermal aging is likely to be important [20]. This issue is however outside the scope of this paper and will be discussed in a future note.

This paper is organized as follows. In section 2, heat transfer and its governing equations will be considered and discussed for metallized film capacitors and subsequently an electrothermal model will be developed. In section 3 the developed thermal model of metallized film capacitors will be used to study the temperature distribution and the likelihood of localized temperature hot spots when the capacitor is subjected to an external voltage. Then in section 4 , temperature distribution is restudied for the heat generated from internal breakdown events in the film capacitor. Numerical examples are used in section 3 and 4 to provide an illustration of the most probable impact under different heat generation conditions in metallised polymer film capacitors. Finally in section 5, observations and conclusions will be summarized.

\section{SIMPLE MODEL OF HEAT TRANSFER IN FILM CAPACITORS}

A metallized polymer film capacitor typically consists of a few thousand flattened polymer layers stacked together and immersed in an impregnation medium in the packaging case unit. As shown in Figure 1, each fattened polymer layer is coated on one side with thin metallic coating. The metallic coating has one of its side edges connected to an electrode terminal and other insulated with side margin that is filled up by the impregnation medium. Side margins are on alternating sides of the stacked polymer layers so that the metallic coatings are connected alternatively to two external electrode terminals. As a first step, we consider a simplified one-layer film capacitor model to allow a one-dimensional simulation of the thermal evolution in film capacitors. Furthermore, we assume that the polymeric films on the top and bottom of the thin metallic coating and the impregnation medium in the winding gap

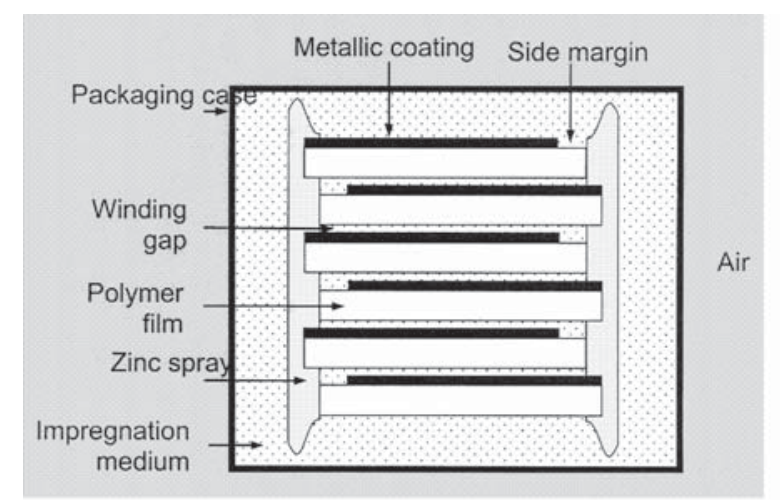

Figure 1. A section of a typical metallized polymer film capacitor. 


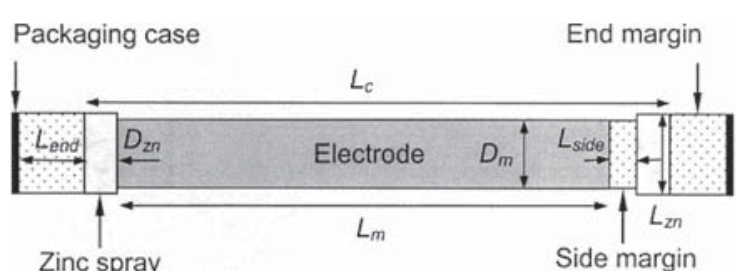

Figure 2. A simplified single-layer film capacitor model for thermal simulation.

are perfect thermal insulators. This assumption will be revisited towards the end of this section. The metallic coating is connected to zinc-spray at both ends with the margin side filled up by impregnation medium. The far end of both zinc-sprays was interfaced with impregnation medium. We also assume that heat flow is directed outwards to air through the vertical plate of the packaging case at the far ends of both impregnation medium sections. The geometrical structure of the simplified capacitor model is illustrated in Figure 2. Zinc is a good conduction material and the length of the zinc spray, $L_{z n}$, is a variable depending on conditions of heat generation. For the heat generation induced by an externally applied electric field, $L_{z n}$ is equal to the length of the two adjacent metallic layers. This is because each layer is impacted by the external field in an exactly the same fashion and so will be assigned to an identical temperature distribution. For numerical examples discussed here, the impregnation medium is assumed to be silicone oil, and temperaturedependence of all materials used in film capacitors are assumed negligible within the timescale of interest. This assumption is reasonable as shown in a stability test to the International Electrotechnique Commission (IEC) requirements [21], whereby the maximum temperature change in the internal and external capacitor unit under the stability test was below $10^{\circ} \mathrm{C}$. A temperature change of $10^{\circ} \mathrm{C}$ in the typical operation temperature range from 20 to $70^{\circ} \mathrm{C}$ does not alter significantly the materials properties used in film capacitors [19, 22]. Ohmic losses induced heat generation can be calculated from the equivalent circuit model of metallised film capacitors illustrated in Figure 3 and detailed in [16, 17]. This facilitates an interface between the heat transfer model and the equivalent circuit based field computation model.

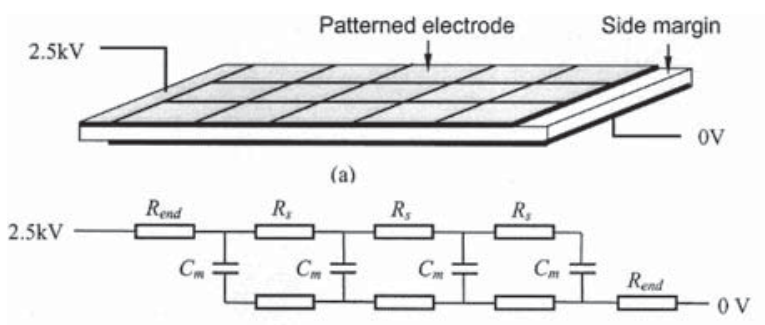

(b)

Figure 3. a, A simplified single-layer film capacitor model; b, equivalent circuit.
In general non-steady heat conduction is governed by [22]

$$
k\left[\frac{\partial^{2} T}{\partial x^{2}}+\frac{\partial^{2} T}{\partial y^{2}}+\frac{\partial^{2} T}{\partial z^{2}}\right]+Q=\rho c_{p} \frac{\partial T}{\partial t}
$$

Here $\rho$ is the mass density in $\mathrm{kg} / \mathrm{m}^{3}, c_{p}$ the specific heat in $\mathrm{J} /(\mathrm{kg} . \mathrm{K}), k$ the thermal conductivity in $\mathrm{W} /(\mathrm{m} . \mathrm{K}), T$ the temperature in $\mathrm{K}$, and $Q$ the internal generated heat per unit volume in $\mathrm{W} / \mathrm{m}^{3}$. Apart from few relatively simple geometrical structures, most heat transfer problems need to be solved numerically rather than analytically. The heat transfer processes in metallized film capacitors are no exception and they need numerical solution. In the one-dimensional limit, equation (1) becomes

$$
k \frac{\partial^{2} T}{\partial x^{2}}+Q=\rho c_{p} \frac{\partial T}{\partial t}
$$

The discretization of the above one-dimensional equation is essentially a simply sequential division along the length of the model capacitor in Figure 2. In total there are $n$ nodes along the model capacitor length. It can be shown that discretization of equation (2) allows temperature at node $i$ at the immediate future instant of time (e.g. at $t_{0}+\Delta t$ ) to be computed from temperature at the current instant of time (e.g. at $t_{0}$ ) via the following formula [22]

$$
T_{i}^{\prime}=T_{i}+\left(\sum_{j} \frac{T_{j}-T_{i}}{R_{i j} C_{i}}+\frac{q_{i}}{C_{i}}\right) \Delta t
$$

where $C_{i}=V_{i} \rho c_{p}$ is the thermal capacitance (in $\mathrm{J} / \mathrm{K}$ ) of the lump volume, $V_{i}$, that surrounds the node $i, q_{i}$ denotes the rate of heat addition to the node $i$ in watts, and $R_{i j}$ is the thermal resistance (in $\mathrm{K} / \mathrm{W}$ ) between the node $i$ and node $j$. The latter two are given by

$$
\begin{gathered}
q_{i}=\frac{T_{i+1}-T_{i-1}}{R_{i+1, i-1}} \\
R_{i j}=\frac{\delta_{i j}}{k A_{k i j}}
\end{gathered}
$$

$\delta_{i j}$ is the distance between the node $i$ and node $j$, and $A_{k i j}$ is the cross sectional area of the lump volume $V_{i}$. Note that the node $i$ and node $j$ are two discretization points along the length of the one-dimensional structure of Figure 4.

Heat transfer processes can be classified into three basic types, namely conduction, convection and radiation [22]. The radiation energy exchange between two surfaces of a finite size area, $A$, is given by [23]

$$
q=A h_{r}\left(T_{1}-T_{2}\right)
$$

where $h_{r}=4 \epsilon \sigma T_{m}^{3}$ is called the radiation heat transfer coefficient. $\sigma$ and $\epsilon$ are the Stefan-Boltzmann constant 


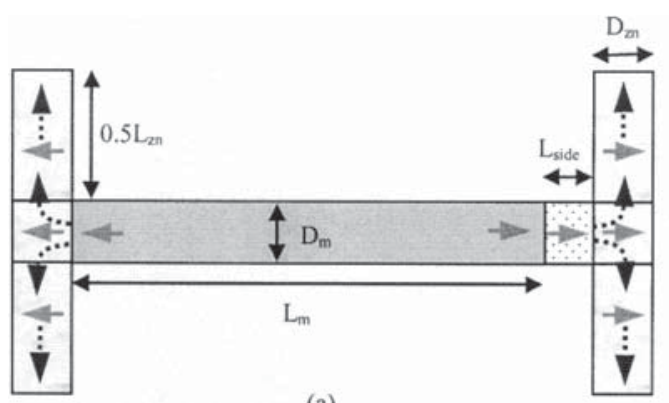

(a)

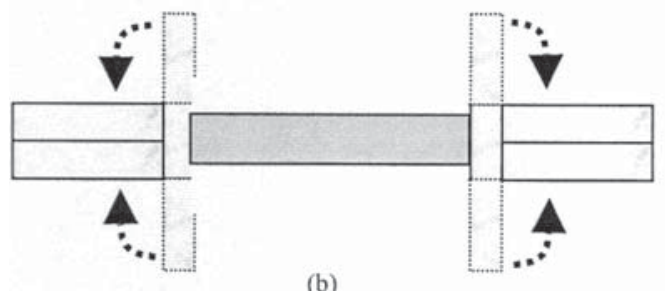

(b)

Figure 4. A simplified thermal model for film capacitors. a, practical structure; $b$, one-dimensional structure.

and emittance of the surface, respectively. $T_{1}$ and $T_{2}$ is temperature for two surface areas. $T_{m}$ is the mean of $T_{1}$ and $T_{2}$. Thermal resistance is defined as the total heat flow through a unit with a thermal potential difference. For conduction, the thermal resistance is given by [22]

$$
R_{k}=\frac{\Delta x}{k A}
$$

where $k$ is the thermal conductivity of a unit material with a distance, $\Delta x$ and a cross section area, $A$. For convection, the thermal resistance is defined as [22]

$$
R_{c}=\frac{1}{h_{r} A}
$$

Using the geometrical dimensions of a typical metallized polymer film capacitor as detailed in section 3 , the thermal resistance due to conduction and radiation can be estimated using equations (5), (6) and (7), and the results are listed in Table 1. The thermal resistance calculation for the heat radiation is based on a blackbody assumption that $\epsilon_{r}$ is equal to 1 . The properties of all materials used in film capacitors at ambient temperature of $20^{\circ} \mathrm{C}$ are based on data in $[22,24]$. Data in Table 1 suggest that the thermal resistance of the $\mathrm{Al}$ film electrode is the lowest, approximately $0.07 \%$ of the polymer section. This justifies our model assumption that the polymer is a perfect ther-

Table 1. Thermal resistance by heat conduction and radiation for different materials.

\begin{tabular}{ccccc}
\hline & \multicolumn{3}{c}{ Conduction } & Radiation \\
\hline Material & $\mathrm{Al}$ & $\mathrm{Zn}$ & $\mathrm{PP}$ & Black body \\
$R(\mathrm{~K} / \mathrm{W})$ & 0.00438 & 0.00892 & 6.25 & 0.1554 \\
\hline
\end{tabular}

mal insulator. Using the geometrical figures typical of film capacitors and assuming the impregnation medium being silicone oil, the thermal resistance of the impregnation layer is found to be 80 times greater than that of the $\mathrm{Al}$ electrode and so similar justification can also be made for the oil section being a perfect thermal insulator. Furthermore we have developed a 2-dimensional computational model to simulate heat transfer in a multi-layer film capacitor and our $2 \mathrm{D}$ results confirm the validity of these assumptions.

The thermal resistance calculation for the heat radiation is an overestimate because of the black body assumption. In practice heat transfer by conduction for polypropylene (PP) and by radiation are likely to be negligible compared with the heat conduction in metallic sections [14]. Based on the empirical relations for free convection in [24] and data in [22, 24], the heat transfer processes by the free convection mechanism for the silicone oil and air in the typical film capacitor is also negligible [14]. As a result, we only consider the heat conduction mechanism in our thermal model. Based on the above assumptions, the thermal model is illustrated in Figure 4a where the solid arrow indicates the heat flow in this onedimensional thermal model. The more realistic heat flow should be that indicated by the dash arrows in Figure 4a. This allows us to modify our thermal model to allow precise heat flow as shown in Figure $4 b$ in which the zinc spray section extended from electrode layer are bent by $90^{\circ}$ to allow heat flow in one-dimension fashion.

\section{HEAT DISSIPATION UNDER EXTERNAL VOLTAGE STRESS}

Based on equation (3) for the thermal model in Figure $4 \mathrm{~b}$ and a computer program is subsequently developed to simulate the evolution of temperature distribution in the single film capacitor. The rate of heat, $q_{i}$, in equation (4) is calculated from the equivalent circuit model of Figure 3 [16]. For numerical examples discussed here, the length of the metallic layer $\left(L_{m}\right)$ and side margin $\left(L_{\text {side }}\right)$ are $96 \mathrm{~mm}$ and $4 \mathrm{~mm}$, respectively. The thickness of the zinc-spray $\left(D_{z n}\right)$ and metallic layer $\left(D_{m}\right)$ are $2 \mathrm{~mm}$ and $10 \mathrm{~nm}$ respectively. The length of the zinc spray is set to the thickness of the polymer film at $7.4 \mu \mathrm{m}$. The surface resistance of the metallic coating is set to $25 \Omega$ [16]. Based on the above parameters, the time constant for the equivalent circuit model is found to be about $1.38 \mu \mathrm{s}$ [16]. The charging time is a small fraction of the discrete time step used in the thermal model. Therefore, the initial temperature distribution of the thermal model was an accumulated result of heat generate from the dc charging process. Furthermore, we assume no additional external or internal heat generation in the thermal model during the transient processes.

During the accelerated life tests of metallized film capacitors, the current is usually limited by a large series- 


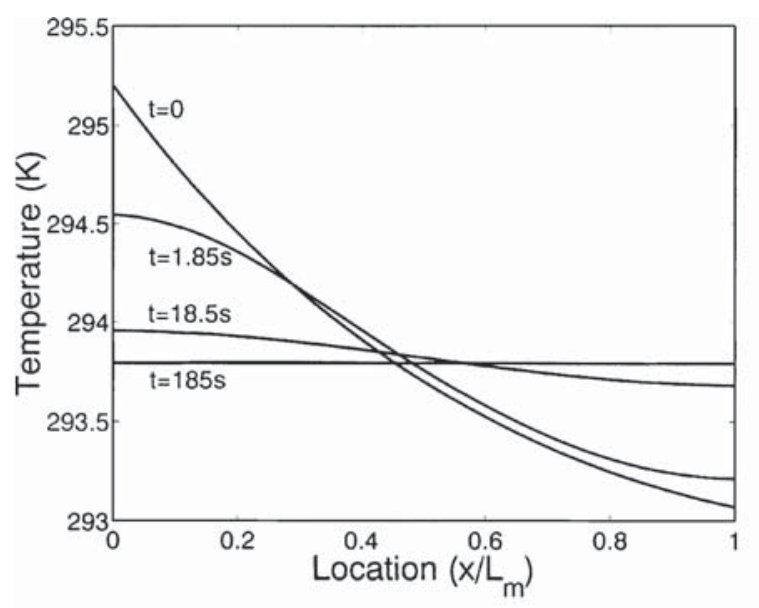

Figure 5. Spatial distribution of temperature along the metallic layer at difference time steps. $\mathrm{x}$ is the distance from the left end of the metallic layer to a point along its length.

connected external resistor to avoid surge current during the charging and discharge processes or disturbance from the external circuit network [21]. The typical current for metallized film capacitors depends on the maximum rate of rise of voltage to which the capacitor may be subjected. In the accelerated life tests conducted in our laboratory, we typically limit the current to below $100 \mathrm{~mA}$, which is limited by the power supply used. Nevertheless, the rate of the rise of voltage in the equivalent circuit model mainly depends on the capacitor parameters. We assume that the current limit $\left(I_{\text {exp }}\right)$ is $100 \mathrm{~mA}$ and the maximum current calculated from the equivalent circuit model is $I_{\text {max }}$. Thus the heat generation from the equivalent circuit model of Figure 3 will need to be divided by $I_{\max } / I_{\text {exp }}$. This is useful for the results calculated from the thermal model to be compared with the experimental results from our accelerated life tests. It is important to note that the magnitude of heat generation does not affect the temperature distribution within the film capacitors, since the non-linear dependence of material properties upon temperature is not considered significant here.

Figure 5 is a plot of the spatial distribution of temperature in the metallic layer at different time steps. Initially the peak temperature occurs at the left-hand edge of the metallic layer through which the majority of electric energy is fed into the system. The peak temperature remains in this area during the transient process before the heat transfer process reaching a steady state at approximately $t$ $=185 \mathrm{~s}$. As a result, the peak average temperature during the entire transient process occurs at the left-hand side of the metallic layer as shown in Figure 6. The localized temperature hot-spot for dc film capacitor is at the edge of the metallic layer connected to the zinc-spray. It is interesting to note that the localized temperature hot-spot can be found directly from the accumulated energy calculated from the equivalent circuit model of metallized film

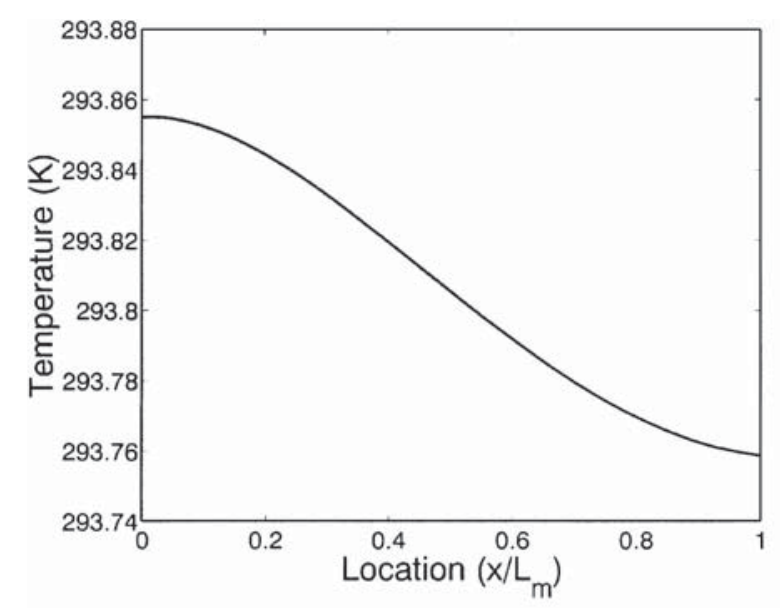

Figure 6. Distribution of the average temperature accumulated along the metallic layer.

capacitors [16]. Also it can be seen that the original temperature of approximately $2^{\circ} \mathrm{C}$ (see Figure 5) is reduced significantly to $0.1 \mathrm{~K}$ (see Figure 6) through dissipation. This indicates that the temperature differentiation due to the application of the external voltage or/and its switching can be largely evened out over a period of about 3 minutes. Therefore localized temperature hot spots are unlikely to be induced by switching of the externally applied voltage stress. This is consistent with the results of our accelerated life tests.

\section{HEAT DISSIPATION UNDER INTERNAL BREAKDOWN EVENTS}

When internal breakdown events extinguish in film capacitors, they deposit significant heat onto the capacitor structure. The heat generated depends on the characteristics of the breakdown events and their occurrence frequency. For cases studied here, we assume that the breakdown events result in a uniform temperature rise of $1 \mathrm{~K}$ across a metallic coating above the ambient temperature of the film capacitor. Our experimental observations are such that breakdown events are rather random and do not have a clear-cut spatial profile. A single breakdown event at a randomly chosen location does not usually lead to any significant heat generation, as confirmed by our numerical simulation and experimental tests. So a uniform profile is the worse scenario in which many breakdowns occur simultaneously across the length of the electrode. Furthermore, we assume the breakdown events are induced in the central metallic layer of the film capacitor. Therefore, the length of the zinc-spray $\left(L_{z n}\right)$ for this case is equal to the thickness of the film capacitor. For practical film capacitors, $L_{z n}=2-5 \mathrm{~cm}$, and the case discussed here we choose $L_{z n}=2 \mathrm{~cm}$.

Figure 7 is a plot of the spatial distribution of temperature on the metallic layer at different time steps for the heat generated by the internal breakdown events. The 


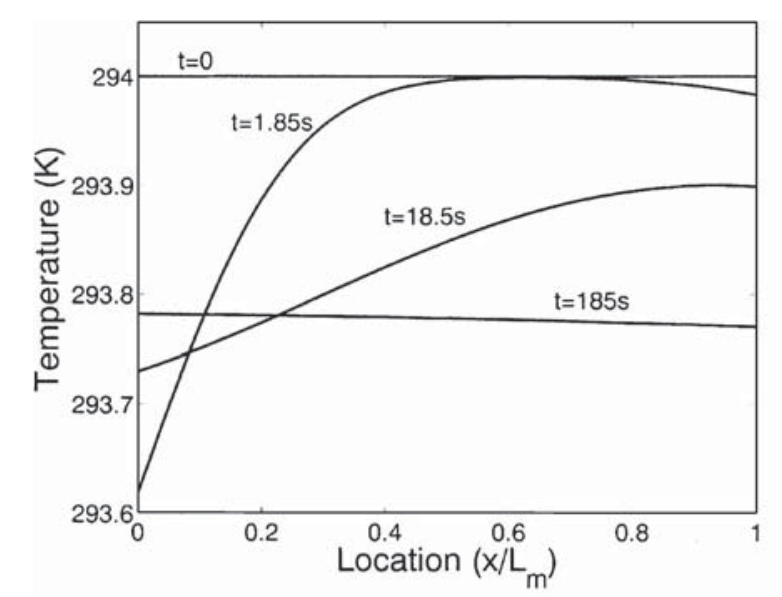

Figure 7. Spatial distribution of temperature along the metallic layer at difference time steps after a spatially uniform internal breakdown event.

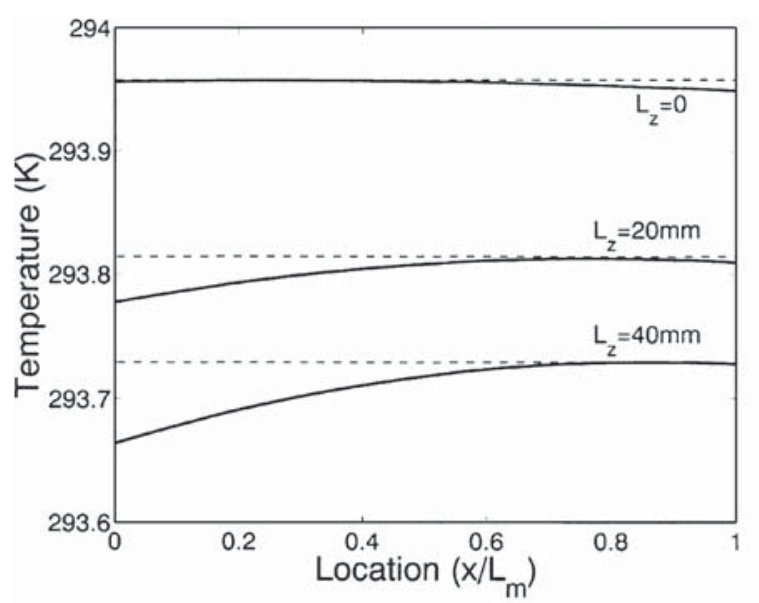

Figure 8. Distribution of the average temperature accumulated during the transient process at difference $L_{z n}$.

peak temperature now moves toward the right-hand side during the transient process before the heat transfer process reaches the steady state at $t=185 \mathrm{~s}$. As a result, the peak average temperature accumulated during the entire transient process is at the right-hand side of the metallic layer as shown in Figure 8. It is interesting to note that $L_{z n}$ mainly depends on the breakdown events. For instance, if breakdowns occur on each metallic layer with the same spatial distribution, $L_{z n}$ would be equal to the distance between the two adjacent metallic layers. For comparison, the average temperature accumulated for $L_{z n}$ being between $0 \mathrm{~mm}$ and $40 \mathrm{~mm}$ is calculated and shown in Figure 8. The results showed that the peak of the localized temperature hot-spot induced by breakdown events is decreased as $L_{z n}$ increases. Nevertheless, the difference between the peak and the lowest temperature becomes larger. It is clear that zinc-spray reacts as a heat sink for heat generated by the extinction of internal breakdown events. More importantly Figure 8 also resembles the spa- tial distribution of broken fuses observed for striped down aged film capacitors [14]. We believe that they are closely correlated and as such one key aging mechanism is the thermal effects of breakdown discharges. This correlation is also found useful to predict the life expectancy of practical film capacitors.

The maximum temperature difference in Figure 8 is about $0.07 \mathrm{~K}$, averaged over $185 \mathrm{~s}$ since an assumed $1 \mathrm{~K}$ temperature rise uniformly along the metallized polymer. This is slightly greater than the $0.1 \mathrm{~K}$ difference in the average temperature induced by an initially $2 \mathrm{~K}$ temperature difference in Figures 5 and 6. In practice an internal breakdown event is likely to be localized and its released energy is also likely to be much greater than indicated by the uniform temperature rise of $1 \mathrm{~K}$. Thus heat dissipation of internal breakdown events is likely to take much longer time, during which subsequent breakdowns may occur. Therefore we envisage that the localized breakdown traces observed from accelerated life tests are mostly likely a result of heat deposition of many microscopic breakdown events that are not necessarily dissipated in an efficient manner in metallized film capacitors. Such multiple and sequential breakdown events will be considered in a future note.

\section{CONCLUDING REMARKS}

An electrothermal model has been developed for metallized film capacitors. Based on a simplified film capacitor model, temperature distribution and localized temperature hot spot have been calculated for different heat generation conditions. It has been established that the localized hot spots can be found from the accumulated energy for metallized film capacitors during the charging processes. For heat generated by the extinction of internal breakdown event cases, the locations and temperature of hot spots mainly depend on the features of the zinc spray and breakdown events. Much of these findings correlate qualitatively to the observation of our accelerated life tests of film capacitors, indicating the applicability of the thermal model developed. Together with the previously developed equivalent circuit model for electric field modelling and the surface discharge model, the thermal model presented here forms the necessary ingredient of a modular global model of metallized film capacitors. This should provide a powerful theoretical tool to unravel the generic features of metallized film capacitors.

\section{REFERENCES}

[1] V. K. Agarwal, "Aging of Multistressed Polymeric Insulators", IEEE Trans. Electr. Insul., Vol. 24, pp. 741-764, 1989.

[2] C. W. Reed and S. W. Cichanowski, "The Fundamentals of Aging in hv Polymer-film Capacitors", IEEE Trans. Dielectr. Electr. Insul., Vol.1, pp. 904-922, 1994.

[3] W. J. Sarjeant, F. W. MacDougall, D. W. Larson, and I. Kohlberg, "Energy Storage Capacitors, Aging, and Diagnostic Approaches for Life Validation", IEEE Trans. Mag., Vol. 33, pp. 501-506, 1997. 
[4] J. L. Nash, "Biaxially Oriented Polypropylene Film in Power Capacitors", Polymer Eng. Sci., Vol. 28, pp. 862-870, 1988.

[5] Y. D. Lee and P. J. Phillips, "The Electrically Ruptured Area of Cross-linked Polyethylene”, IEEE Trans. Electr. Insul., Vol. 26, pp. 171-177, 1991.

[6] B. Sanden and E. Ildstad, "DC Electrical and Mechanical Characterisation of Polypropylene Film", IEEE Intern. Conf. Conduction Breakdown in Solid Dielectrics, Vasteras, Sweden, pp. 210-213, 1998.

[7] A. Gadoum, B. Gosse, and J-P Gosse, "Accelerated ac Degradation of Impregnated PP Films", IEEE Trans. Dielectr. Electr. Insul., Vol. 2, pp. 1075-1082, 1995.

[8] A. Schneuwly, P. Groning, and L. Schlapbach, C. Irrgang, and J. Vogt, "Breakdown Behaviours of Oil-impregnated Polypropylene as Dielectric in Film Capacitors", IEEE Trans. Dielectr. Electr. Insul., Vol. 5, pp. 862-868, 1998.

[9] N. Berger and P. Jay, "Impregnant-polypropylene Interactions in the All-film Capacitor Configuration", IEEE Trans. Electr. Insul., Vol. 22, pp. 383-388, 1987.

[10] A. Gadoum, B. Gosse, and J. P. Gosse, "Effect of An Epoxy Additive on the Electrical Aging of Impregnated Polypropylene Films", J. Appl. Polymer Sci., Vol. 62, pp. 1679-1692, 1996.

[11] K. Uchida and N. Shimizu, "The Effect of Temperature and Voltage on Polymer-chain Scission in High-field Region”, IEEE Trans. Electr. Insul., Vol. 26, pp. 271-277, 1991.

[12] J. R. Laghari, "High-voltage Pulsed Life of Multistressed Polypropylene Capacitor Dielectric", IEEE Trans. Nuclear Sci., Vol. 39, pp. 21-24, 1992.

[13] A. Borghetti, C. A. Nucci, G. Pasini, S. Pirani, and M. Rinaldi, "Tests on Self-healing Metallized Polypropylene Capacitors for Power Applications", IEEE Trans. Power Delivery, Vol. 10, pp. 556-561, 1995.

[14] Y. P. Lee, Electrically and thermally activated aging mechanisms in metallised polymer film capacitors, Ph.D. Thesis, Loughborough University, UK, 2001.

[15] Ch. Joubert, A. Beroual, and G. Rojat, "Electric Field and Equivalent Circuit in All-film Capacitor", J. Appl. Phys., Vol. 81, pp. 6579-6584, 1997.

[16] Y. P. Lee and M. G. Kong, "Surface Field Dynamics in dc Film Capacitors Under an Impulse Voltage Perturbation", IEEE Trans. Dielectr. Electr. Insul., Vol. 8, pp. 293-298, 2001.

[17] Y. P. Lee and M. G. Kong, "Numerically Established Correlation in Electrical Responses of Polymer Film Capacitors to ac and Pulsed Voltage Perturbation”, IEEE Trans. Dielectr. Electr. Insul., Vol. 8, pp. 880-888, 2001.
[18] J. Kammermaier, G. Rittmayer, and S. Birkle, "Modelling of Plasma Induced Self-healing in Organic Dielectrics", J. Appl. Phys., Vol. 66, pp. 1594-1609, 1989.

[19] M. G. Kong and Y. P. Lee, "Impact of Surface Discharge Plasmas on Performance of a Metallized Film Capacitor", J. Appl. Phys., Vol. 90, pp. 3060-3078, 2001.

[20] M. A. Bagirov, M. A. Kurbanov, and E. A. Garagashev, "Destruction of Polymer Dielectrics by Partial Discharges", Sov. Phys. Tech. Phys, Vol. 20, pp. 55-57, 1975.

[21] International Electrotechnical Commission: Power Electronics Capacitors, CEI/IEC 1071-1, 1991.

[22] A. J. Chapman, Heat Transfer, $3^{\text {rd }}$ edition, Macmillan, New York, 1974.

[23] A. F. Mills, Heat Transfer, $2^{\text {nd }}$ edition, Prentice-Hall, New Jersey, 1999.

[24] J. P. Holman, Heat Transfer, $8^{\text {th }}$ edition, McGraw-Hill, New York, 1997.

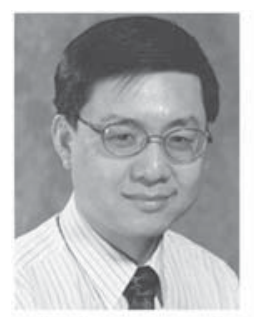

Michael G. Kong (M'94-SM'98) received the B.Sc. and M.Sc. degrees, in electronics engineering, from Zhejiang University, China, in 1984 and 1987, respectively, and the Ph.D. in electrical engineering from Liverpool University, UK, in 1992. He was appointed in 1995 a Lecturer at Liverpool University and in 1999 to a Senior Lecturer at Loughborough University, UK where he is now a Professor of Bioelectrics. He has been involved in research on nonthermal atmospheric gas discharges, compact waveguide free electron lasers, and power systems and devices. In these subject areas, he has published some 100 papers in scientific journals and conference proceedings.

Yuen Pen Lee received the B.Sc. degree in electrical engineering from Liverpool University in 1997 and the Ph.D. degree also in electrical engineering from Loughborough University in 2001. He is an Associate Member of IEE. His research interests are electrical insulation and power electronics systems. 